\title{
Chercheurs de « L'entre-DeUX » - Travailleurs SOCIAUX DANS LE RÔLE DE CHERCHEURS : PROXIMITÉ ET DISTANCE D’UNE ETHNOGRAPHIE INTERPRÉTATIVE ${ }^{\star}$ http://dx.doi.org/10.1590/1984-0292/1334
}

\section{Somnoma Valérie Ouédraogo $\star \star$ Université d'Ottawa, Ottawa, Canadá}

\begin{abstract}
RÉSUMÉ
Le présent article porte sur les réflexions de terrain menées au Burkina Faso dans le cadre d'une étude doctorale qui a porté sur le retour forcé de travailleurs migrants burkinabè. Il interpelle à la fois les chercheurs de "l'entre-deux » et les chercheurs en travail social qui se trouvent pris à porter des chapeaux parfois en tension en tant que personne appartenant à la société étudiée, praticiens et chercheurs. L'objectif de notre article se resserre à cet effet autour des deux points c'est-à-dire chercheuse de l'entre-deux et travailleuse sociale placée dans le rôle de chercheuse avec le terrain.

Mots-clés : ethnographie ; chercheurs de l'entre-deux; chercheurs-praticiens.

\section{RESEARCHERS OF THE "IN-BETWEEN" - SOCIAL WORKERS IN THE ROLE OF RESEARCHERS: PROXIMITY AND DISTANCE}

\section{OF AN INTERPRETATIVE ETHNOGRAPHY}

\begin{abstract}
This article focuses on the reflections of a fieldwork conducted in Burkina Faso as part of a doctoral study that focused on the forced return of Burkinabè migrant workers. It speaks to both researchers 'in-between' and researchers in social work who find themselves caught wearing hats sometimes in tension as practitioners, researchers and none the less as fieldworkers who belong to the studied society. The purpose of this article to that effect tightens around the two points that means the positions of researchers "in-between" and social workers placed in the role of researcher during interactions in field.

Keywords: ethnography; researchers of "in-between"; researchers-practitioners.

^ Cet article s'inspire du contenu du chapitre 4 de ma thèse de doctorat (OUEDRAOGO, 2013, p. 86-108) publiée en Allemand. Les données utilisées dans le cadre de cet article sont les notes de terrains, les protocoles d'observation, les entretiens et les protocoles de réflexion de terrain élaborés lors des échanges, des expériences de terrain avec ma superviseure et majoritairement avec mes informateurs. L'alternance entre proximité et distance dans le cas de cette étude répond plus à une logique de dialogue dans le processus de constitution de notre corpus de données. Le projet de doctorat a été finance par une bourse de la fondation Friedrich-Ebert.

$\star \star$ Professeure à la leçon, École de Service Social, Université d'Ottawa 120 Université, Pavillon des sciences sociales, Ottawa ON K1N6N5, Pièce 12043. courriel: vouedrao@uottawa.ca
\end{abstract}




\section{INTRODUCTION}

En avril 2008 j'ai présenté un exposé sur les migrations de travail entre le Burkina Faso et la Côte d'Ivoire dans le cadre de la rencontre annuelle des boursiers étrangers de la fondation Friedrich-Ebert dans la région de Bonn. ${ }^{1} \mathrm{Je}$ suis parti de la perspective économique push-pull (LEE, 1966, p. 47-57) pour montrer les facteurs d'inégalités économiques qui sous-tendent les mouvements migratoires entre les deux pays. Le Burkina Faso étant le pays de départ c'est plus la question des effets économiques et sociodémographiques (OUEDRAOGO ; PICHÉ, 2007; PACÉRÉ, 2004; MANDÉ, 2003) de la migration que montraient les études sur lesquelles je me suis basée pour élaborer mon exposé. Étant en fin de cycle de mes études en travail social je menais pendant ce même temps des recherches sur l'intégration des travailleurs immigrants dans l'état de la Saxe en Allemagne. Dans le cadre de la rédaction de ma thèse de diplôme les discussions menées lors de mon exposé continuaient de temps à autre à surgir dans mes réflexions sur la notion de l'intégration dans le pays d'accueil. Partant donc de ma thèse de diplôme il s'est avéré pour moi pertinent de mener une étude sur l'intégration des travailleurs migrants burkinabè qui, à cause de la crise sociopolitique en Côte d'Ivoire, ont été contraints en 2002 au retour dans leur pays d'origine. La pertinence de ce retour forcé en tant que phénomène social est fondée sur deux raisons: la première est que le Burkina Faso compte tenu de ce retour forcé a basculé de son symbole de pays de départ à un symbole de pays d'accueil. D'ou l'intérêt de questionner le processus d'intégration de ces nouveaux arrivants dans la société d'accueil. La deuxième raison tient compte du temps entre le retour en 2002 et le projet de doctorat qui comme je l'ai mentionné commençait à germer en 2008. Vu que cela faisait plus de cinq ans que les travailleurs migrants sont de retour la probabilité était qu'ils étaient peut-être retournés de nouveau en Côte d'Ivoire. L'enquête de terrain de type ethnographique s'avérait donc la méthode appropriée pour investiguer à ce sujet : où sont-ils? Que font-ils? Comment organisent-ils leur vie quotidienne? Et comment surmontent-ils leur ré-migration? ${ }^{2}$ Autrement dit l'étude devrait procéder par identifier la localisation des sujets aller à leurs rencontre puis progressivement procéder au recrutement des informateurs pour les interviews (BEAUD; WEBER, 1998). Suite à mon diplôme de travailleuse/pédagogue sociale j'ai donc entamé des études doctorales sur la ré-migration de travailleurs migrants burkinabè. Mon doctorat s'est déroulé au département de travail social à la faculté des sciences humaines de l'université de Kassel en Allemagne. Partant donc de l'approche ethnographique de notre étude de terrain nous avions avant d'entrer sur le terrain au Burkina Faso l'idée de centrer l'étude sur l'expérience des ré-migrants en tant que sujets ayant vécu ce retour. Les analyses politiques, économiques et sociodémographiques (rapports des ministères, de l'organisation internationale de la migration, résultats d'études de politologues, sociologues et démographes) sur la thématique étaient censées servir comme informations complémentaires pour mieux comprendre le contexte macro social de la ré-migration. Nous n'avions pas un cadre théorique défini en tant que tel, mais l'hypothèse que compte tenu de la situation de crise dans leur pays d'accueil les ré-migrants ont été contraints « en tant que Burkinabè » de 
retourner dans leur pays d'origine. À cet effet, nous supposions que les pistes empiriques nous conduiront peut-être à nous pencher sur des théories portant sur les appartenances nationales. Partant de ce postulat nous avons privilégié un cadre interprétatif devant nous permettre de mobiliser les analyses théoriques appropriées au corpus de données empiriques (GLASER ; STRAUSS, 2010). La recherche a été à cet effet effectuée afin de comprendre la ré-migration à travers la perspective des ré-migrants eux-mêmes. Les études de terrain ont été menées en deux phases : la première en 2009 pendant quatre mois et la seconde en 2010 pendant deux mois. Le terrain s'est déroulé dans deux lieux d'observation que nous avons dénommés dans le cadre de notre étude quartier 62 et $K$-dorf. Les ré-migrantes étaient constituées sous la forme d'une association au quartier 62 où était le siège de leur association Stern. ${ }^{3}$ Mais également la plupart des membres de l'association habitaient le quartier 62. Le groupe des ré-migrants et ré-migrantes de $K$-dorf n'était pas organisé comme une association, mais comme des individus ${ }^{4}$ appartenant à plusieurs familles. La forme d'observation et de participation à laquelle nous avions fait recours est l'observation non structurée dont le principe fondamentale selon Roland Girtler (2001) requiert une cumulation simultanée des deux fonctions et rôles en tant qu'observatrice et participante (Feldforscherin). Tout au long de mon terrain je me suis présentée tout d'abord comme une étudiante. Au fur et à mesure lorsque les acteurs sur le terrain ont voulu lors de nos conversations savoir si j'étudiais à l'université de Ouagadougou. J'ai répondu en disant que j'étudiais en Allemagne. Sans pour autant mentionner mon statut académique de travailleuse sociale. J'étais donc sur le terrain en tant qu'étudiante et non en tant que stagiaire en travail social.

\section{Chercheuse de l'entre-deuX - travailleuse sociale dans le} RÔLE DE CHERCHEUSE

$\mathrm{Au}$ Burkina Faso lorsqu'une personne de votre réseau social vous reproche de ne plus avoir de vos nouvelles, la personne mise en cause répond le plus souvent « qu'elle ne fait que reculer pour mieux sauter. « De notre point de vue, cette phrase met en scène, un mouvement de recul et d'avancement qui interpelle des acteurs à une interaction. Autrement dit les acteurs sont appelés à tisser, établir et entretenir une relation. Le recul et l'avancement sont entrepris à travers les pas qui se régulent, les yeux qui scrutent, la tête qui calcule autrement dit à travers l'image du corps et des émotions qui se mettent en relation et en action pour jauger comment reculer pour mieux sauter. Il s'agit d'une action qui met en exergue des personnes qui interagissent pour donner place et existence à une relation (avec de multiples degrés de participation et d'attente). C'est une analogie que je transpose sur le terrain de recherche pour décrire et analyser les notions de proximité et de distance qui articulent l'interaction in situ (aussi bien au quartier 62 qu'à K-dorf ) à la fois de la chercheuse de l'entre-deux et la travailleuse sociale dans son rôle de chercheuse avec les ré-migrants. La dyade reculer et mieux sauter représente la relation entre proximité et distance dans la réalisation de l'observation participante avec le terrain. Mon hypothèse est que de cette relation de dyade, il résulte des tensions selon les attentes que chacun des acteurs 
(chercheuse et ré-migrants) vise dans la proximité et la distance. Partant d'une telle thèse, j'emprunterai l'approche anthropologique d'Abu-Lughod pour penser les moments de distance opérés au quartier 62 et à K-dorf lors de notre étude. L'approche d'Abu-Lughod (2010), tout comme celui de Girtler (2001), interpelle la chercheuse à adopter une perspective interprétative pour étudier les relations entre les acteurs en situation d'interdépendance tenant compte des situations in situ. Abu-Lughod met au centre de sa réflexion la question de positionnement et de partialité dans la flexibilité de chercheurs de terrain qui empruntent la méthode d'observation participante pour étudier un phénomène social au sein de leur propre société. Ces chercheuses de terrain que Abu-Lughod nomme hybrides sont des chercheuses qui ont étudié et/ou élu domicile dans un pays autre que leur pays d'origine, et qui ont acquis une expérience migratoire et se retrouvent dans une relation de "l'entre-deux ». Réfléchir sur les positionnements de la chercheuse hybride sous l'angle de l'entre-deux c'est analyser « qui parle à la place de qui et en tant que qui ? »(ABU-LUGHOD, 2010, p. 426) quand il s'agit de se positionner dans une interaction sur le terrain. Cela signifie que les décisions et stratégies prises dans l'interaction reposent certes sur les pistes adéquates pour la constitution de l'échantillon requis, mais interpelle simultanément la chercheuse à interroger sa propre hybridité. Ce qui sous-tend le questionnement de son entredeux concernant les décisions, stratégies et positionnements attribués ou mis en interaction avec le groupe social étudié pour construire la réalité sociale qu'on entreprend d'analyser. La déconstruction de son ethnocentrisme (SUMNER, 1906) en lien avec l'observation participante consiste donc à prendre son entre-deux non pas seulement comme une limite qu' on fait semblant de ne pas saisir, mais de se l'approprier comme une possibilité qui pourrait favoriser la production de connaissance si on met cette possibilité en réflexivité. L'hypothèse qui sous-tend l'entre-deux selon Abu-Lughod dans la même ligne de pensée de la flexibilité que recommande l'approche de Girtler, est que l'hybridité de la chercheuse est partie prenante des moments de distance dans la constitution in situ de l'échantillon. Cette hybridité se manifeste selon l'union de rôles tenant compte des contextes, des situations et de la flexibilité avec lesquels les «frames » (GOFFMAN, 1991) de l'entre-deux et/ou de la biographie identitaire interagiront avec le terrain.

\section{II.1 L'hybridité de l'entre-deux - biographie identitaire avec le terrain}

Vu sous cet angle d'analyse, mon d'hybridité est composée entre autres premièrement de mon expérience de vie en tant que Burkinabé au Burkina Faso et mon expérience migratoire en tant qu'étudiante étrangère en Allemagne. ${ }^{5}$ Issue d'une société avec une expérience collective coloniale j'ai dû me confronter par moment au fait d'être noire, femme et Africaine subsaharienne dans une Allemagne du XXIe siècle. Autrement dit les " frames » reflétant ma propre expérience migratoire et les discriminations vécues dans le cadre de cette expérience. À ces premiers cadrages j'ajoute deuxièmement mon expérience d'une part linguistique qui fait de moi un hybride comprenant au moins quatre langues (moré, allemand, français, anglais), d'autre part académique qui fait de moi un hybride formé en tant que travailleuse/pédagogue sociale et chercheuse. Comme le souligne AbuLughod l'ethnographie est une méthode qui définit la relation entre l'occident 
et le non-occident. Et cette relation est la domination occidentale (ABULUGHOD, 2010). Dans ces cadrages de la domination occidentale je résume des positionnements quant à la question de la relation d'aide. Cette relation d'aide caractérise, selon ma perspective, autant les services sociaux (travail social), que les rapports entre migrants et sociétés d'accueil (migrations) et sans oublié les rapports entre sociétés ex-colonisées et ex-colons (postcolonialisme). D'où la pertinence d'analyser les tensions sous-jacentes des moments de proximité et de distance à la fois de la chercheuse de l'entre-deux et la chercheuse en travail social. Pour résumer la perspective des éléments requis pour la flexibilité dans la pratique de l'observation participante, je dirais que ces différents cadrages de mon hybridité constituent un ensemble de savoirs-être et savoir-faire autrement dit de postulats puis de potentialités d'interaction avec le terrain étudié. Et ses savoirs-être et savoir-faire sont des postulats et des potentialités dont sont dotés mes interlocuteurs également. En tant que des acteurs nous constituons des êtres dont les corps et les mots sont inscrits d'expériences historiques, biographiques et intellectuelles (migration, éducation, femmes, hommes, Burkinabè, etc.). Dans ce cas comment se résolvent ces crises au cours de l'observation participante ? Quelle est la distance qu'il faut pour entretenir la proximité requise dans une telle relation? Jusqu'à quel point maintenir la distance sans pour autant mettre fin à la proximité que l'on est parvenu à mettre en place et en action, en tant qu'acteurs?

\section{II.2 Contact avec le terrain - entrée dans le terrain}

Pour Girtler (2001) la proximité est une nécessité pour que la chercheuse de terrain puisse s'identifier avec son terrain, afin de pouvoir cerner son objet de recherche. Alors que la distance est un retrait momentané hors du terrain, dans le but de réfléchir à la proximité nécessaire pour la constitution du corpus de recherche. Ce processus de proximité se déroule, selon Weinberg et Williams, à travers différentes phases qui ne sont pas linéaires. Ces phases selon les deux auteurs sont: le rapprochement (Annährung), l'orientation (Orientierung), l'initiation (Initiation) et l'assimilation (Assimilation) (WEINBERG ; WILLIAMS, 1973, p. 83-108).

Dans le cas de la présente étude le rapprochement, autrement dit le moment d'établissement de relations avec le groupe social étudié s'est réalisé à travers les démarches entreprises par la chercheuse de terrain pour prendre contact avec les ré-migrants aussi bien au quartier 62 qu'à $K$-dorf. Cette phase s'est manifestée concrètement pour la chercheuse de terrain une fois que les personnes contactées ont accepté qu'elle mène son étude en participant à leur quotidien (Note de terrain, août 2009).

L'orientation est la phase où la confiance et l'implication de la chercheuse de terrain commencent à s'établir dans les interactions sociales étudiées (GIRTLER, 2001, p. 93). Par exemple dans le cas de notre étude, Samuel, un des ré-migrants de K-dorf, s'est porté volontaire pour me conduire aux domiciles des autres ré-migrants qui à K-dorf, ont acceptés de participer à l'étude. Ce jour-là nous n'avions trouvé personne à son domicile. Samuel me dit qu'ils sont sûrement dans leurs champs agricoles. Il poursuivit que lui-même a abandonné la récolte des arachides à sa femme pour venir comme promis m'apporter son soutien. Puis 
nous sommes allés chez lui et il me présenta sa mère. En route il s'arrêtait à chaque fois pour saluer des passants, me les présentait en disant à ces derniers, qu'il me montre les chemins menant facilement aux domiciles des personnes qui ont accepté de participer à mon étude. Il me montra les chemins, m'indiquant les plus courts, les plus longs, ceux qui déboucheront plutôt sur des marécages, etc. Il me raconta que K-dorf est divisé. Les fonctionnaires, continua-t-il, se distinguent des habitants, ne voulant pas se fondre à eux. De la même manière, même si les femmes de ces fonctionnaires sont toutes aussi bien des ménagères que les femmes des habitants, les premières sont persuadées qu'elles sont mieux loties par rapport aux autres femmes qui vivent à K-dorf. Je l'écoutais tout en observant l'environnement. Je portais attentions aux chemins, mais aussi à la différence entre la zone lotie et la zone non lotie de K-dorf. Alors que la zone lotie abrite des villas luxueuses, on constate que la zone non lotie, où nous étions, regroupait des champs et des maisons simples. Les chemins étaient étroits et, en cette saison pluvieuse, ils étaient difficiles à emprunter.

Le récit de Samuel me permettait de m'orienter, mais également m'offrait des informations sur les différents groupes sociaux qui sont à K-dorf. Nous pouvons donc constater que la confiance requise lors de la deuxième phase s'établit à travers l'orientation géographique, mais aussi grâce aux informations sur l'environnement d'interaction du groupe cible. Ce sont ces informations qui représentent les savoirs dits directement liés à l'objet de recherche (GARNEAU, 2012, p. 143-145). Le contact in situ avec le groupe dans son environnement social (dont l'étude est concernée) permet à la chercheuse de terrain de commencer à formuler des pistes d'investigation. À travers mon exemple, on peut formuler comme piste l'hypothèse que la chercheuse de terrain construit selon sa perception et son vécu in situ (les chemins, les maisons, les gens rencontrés) et les récits de Samuel des suppositions sur les classes sociales à K-dorf c'est-à-dire les fonctionnaires et les autres. Quant à la relation chercheuse de terrain et ré-migrant, l'interaction se déroulait entre les récits de Samuel dans son rôle d'expert et la chercheuse de terrain dans son rôle de la chercheuse acceptée, à qui Samuel inculquait certaines notions d'orientation de son nouveau monde d'adoption.

Comme troisième phase nous avons l'initiation qui représente la relative implication de la chercheuse avec son nouveau monde social qui l'accepte. Dans le cas de notre étude, cette phase d'initiation s'est manifestée par exemple au quartier 62 à travers les entretiens que la chercheuse a eu avec le conseiller et la présidente de l'association Stern pour mieux connaître l'histoire, les objectifs et les activités de leur association. Suite à ces deux entretiens, elle a pu se rendre compte des composantes sociales de l'environnement socioéconomique des ré-migrantes $\mathrm{du}$ quartier 62. Cette phase d'initiation m'a permis premièrement de prendre en considération la présence et l'importance d'autres acteurs à prendre en compte dans l'étude. Deuxièmement la phase d'initiation ouvre d'autres perspectives pour cerner les aspects pertinents de ré-migration que l'étude devrait prendre en considération dans son corpus d'analyse. D'où la prise de conscience de la distinction des diverses formes sociales chez les ré-migrants, c'est-à-dire association, individus et familles qui permettent d'adapter les situations de recherche. C'est donc l'entretien auprès 
des deux responsables de l'association qui ont initié la chercheuse à son nouveau monde. L'association a été recrutée pendant le terrain lorsque la chercheuse était à la recherche d'éventuels ré-migrants restés au Burkina Faso. C'est par l'intermédiaire d'une personne travaillant au ministère de l'action sociale que $\mathrm{j}$ 'ai entrepris mes contacts initiaux auprès de Marie. L'intermédiaire me disait qu'elle était une rémigrante et qu'à travers elle je pourrais avoir la chance de rencontrer d'autres rémigrants pour mon étude. En effet, c'est lors de notre première rencontre que Marie m'informa de l'existence de l'association Stern dont elle était la présidente. Ce qui pour l'étude permettait de valider que certains ré-migrants étaient restés et d'autres (comme Marie) tentaient de se regrouper en association pour organiser des activités génératrices de revenu. L'association en tant qu'organisation sociale des femmes ré-migrantes s'est donc avérée selon les aléas du terrain comme une possibilité d'entrée en relation avec le groupe cible de l'étude. Nous pouvons partant de nos exemples constater que les phases d'entrée dans le terrain ne sont pas des étapes linéaires mais un processus chevauchant entre renseignement, investigation, localisation, rencontre, entente etc.

Les trois premières phases (rapprochement, orientation et initiation) permettent donc à la chercheuse de terrain de cerner et de prendre place dans les lieux et les interactions possibles concernés par l'étude. C'est lors de ces processus que se construisent les hypothèses, les suppositions, les pistes possibles qui sont à prendre en compte lors de l'étude (BECKER, 2003). C'est également lors de ce processus que la chercheuse fait les choix et prend les décisions pour délimiter l'échantillon qui devra au fur et à mesure être constitué dans sa spécificité et dans son ensemble pour permettre de disséquer le phénomène étudié.

La quatrième phase qui est l'assimilation représente la familiarité de la chercheuse avec son terrain c'est-à-dire les lieux, les acteurs, les thèmes qui les préoccupent et les interactions qui in situ en régissent le quotidien. C'est la phase qui favorise l'interdépendance (LÉVI-STRAUSS, 1955) permettant aux acteurs de construire leur réalité sociale. L'assimilation est donc source d'une interaction se conformant aux valeurs, aux attentes etc. du monde social observé et vécu (GEERTZ, 2003). Autrement dit autant la chercheuse de terrain que le groupe social observé en viennent à mettre leur code de communication, leurs attentes et leur vision du monde en action. D'où le sentiment de vies bien réelles, ancrées dans une construction sociale partagée les uns avec les autres (GOULET, 2011).

\section{II.3 Proximité avec le terrain - la réalisation des relations}

Suite à l'assimilation la chercheuse de terrain devient un membre à part entière auquel le groupe social étudié attribue des positions. Ces derniers se conduisent envers elle en conséquence, c'est-à-dire comme membre ayant une place et des positions à remplir (FLAMANT, 2005, p. 138-140). Cette familiarité fait donc qu'elle s'implique ou, est de plus en plus, invitée à s'impliquer dans les interactions qui régissent le social du groupe observé. D'où le danger que la chercheuse de terrain se mette à penser et à agir à la place de certains membres du groupe social étudié. Il peut en résulter une distorsion de l'observation, car à cette étape la chercheuse de terrain croit mieux comprendre le monde social 
étudié et agit en conséquence. Ce qui l'emmène à calculer, prévoir et anticiper parfois les interactions. Ces moments de déformations sont vus comme des limites de la méthode d'observation participante. Ces limites peuvent par exemple selon Girtler se manifester comme un " going native » (GIRTLER, 2001, p. 78-82). Ce phénomène se définit comme une identification extrême des actions de la chercheuse avec les interactions étudiées. Et ce au point de perdre en vue le fil du processus de recherche, les objectifs de l'étude menée et le postulat d'objectivité requis pour une recherche scientifique. La chercheuse dans ses moments d'identification extrême se trouve dans une position de routine ce qui la conduit à s'approprier des règles et codes sociaux étudiés comme siens. Elle devient donc membre à part entière du processus social observé et fait de l'angle de ses propres perspectives celui du groupe social étudié. Un autre danger du « going native » est que cette extrême identification conduit progressivement à rendre flou la curiosité qui, en ethnographie, se révèle comme étant l'élément générateur qui entretient le mythe de l'inconnu. C'est cet inconnu dont on se met à la rencontre pour comprendre la spécificité et l'ensemble qui nourrit son étrangeté (son côté non encore étudié).

Par exemple dans le cas de notre étude, la chercheuse prit l'initiative de discuter avec les ré-migrantes du quartier 62 à la suite d'une insatisfaction vis-àvis des positions qu'elle remplissait :

Je viens d'envoyer un courriel à ma superviseure, car j'ai comme l'intuition que c'est plus l'âme de la travailleuse sociale que l'âme de la chercheuse qui interagit et cela perdure même si au fond je veux juste comprendre sans pour autant avoir à trouver des solutions aux problèmes au quotidien ; qu'est-ce que j'ai ? Qu'est-ce que je cherche? Qu'est-ce que je veux ? (Journal de recherche, 05 octobre 2009).

Après avoir discuté avec ma superviseure du contenu de mon courriel, je pris le temps d'expliquer aux ré-migrantes lors de la tenue de leur assemblée générale mensuelle que je voulais bien continuer à rédiger leurs lettres administratives, les accompagner auprès de l'administration pour résoudre les tâches administratives de leur association, mais que je ne voulais pas continuer à prendre des décisions pour elles. J'ajoutai ce jour-là qu'elles-mêmes ainsi que leur conseiller pouvaient continuer à bien mieux diriger les décisions que moi parce qu'ils sont les initiateurs et les visionnaires de leur association. Puis je conclus en leur disant que j'espérais qu'elles continueraient à participer à mon étude. Certaines ré-migrantes prirent la parole à tour de rôle pour dire que selon elles j'étais là pour étudier l'organisation et le travail de leur association. D'autres continuèrent en disant que d'habitude les étudiants s'intéressent à la participation de la femme dans la société. D'autres encore insistaient en disant que j'avais bien dit que je voulais savoir comment était leur vie en Côte d'Ivoire. C'est suite à leurs interventions orales que je réalisais in situ qu'elles avaient (en tant que « femmes » et grâce à leur association) l'expérience d'être une population enquêtée pour différents buts d'études. Pendant que je ruminais en moi comment leur dire que je m'intéressais plus aux histoires de leurs vies au Burkina Faso 
depuis leur retour de la Côte d'Ivoire sans pour autant que cela suppose « je ne m'intéresse pas à votre association ", la présidente, Marie, me proposa séance tenante d'établir des contacts individuels avec celles qui seront intéressées à participer à mon étude. Elle conclut en disant que je pouvais rester. Toutes applaudirent cette décision finale et l'assemblée prit fin là-dessus.

Après cet aveu lors de l'assemblée j'étais, en plus des activités administratives de l'association, informée sur les évènements qui touchaient le quotidien des ré-migrantes au quartier 62 . Je fus tenu de rencontre pour régler les conflits entre membres. Je fus mise au courant des naissances, des décès, des maladies et des difficultés avec les membres des familles qui les avaient hébergés. Aussi hors mis le siège de l'association les ré-migrantes qui le voulaient me recevaient quotidiennement chez elles pour mes activités de recherche. Il y a donc eu un moment de recul qui s'est manifesté à travers le courriel, les réflexions menées avec la superviseure et la discussion avec les acteurs de terrain. Comme relaté ci-dessus la discussion a appelé une reconsidération des attentes vis-à-vis les unes des autres. Le rôle de l'experte en administration m'a permis de mieux cerner l'histoire et l'évolution de l'association. Mais ce rôle ne me permettait pas d'avoir les éléments nécessaires pour faire le lien entre l'association et la ré-migration qu'elles ont vécu et continue de vivre au quotidien. La chercheuse avait ainsi la possibilité d'agencer de nouveau (et à chaque fois que cela s'avérait nécessaire) cette proximité individuellement avec chacune des ré-migrantes.

Les crises se résolvaient donc par le fait que des personnes ressources étaient disposées pour parler des expériences de terrain perçues comme telles par la chercheuse. Aussi les ré-migrants étaient ouverts pour réajuster les interactions en lien avec mes rôles apparemment fixés. Tant que la chercheuse se confinait à garder le rôle de l'experte en possession de connaissances administratives les ré-migrantes l'impliquaient davantage dans les évènements économiques de l'association. Dans ce rôle il lui était impossible de s'entretenir avec elles des événements en rapport avec leur ré-migration, de la situation qu'elles vivent suite à ce retour, des expériences qu'elles ont vécues en Côte d'Ivoire et celles qu'elles vivent au Burkina Faso, etc. Cela lui a permis au fur et à mesure d'appréhender leur quotidien, de s'entretenir avec elles pas sur des chiffres de vente de leur attiéké, mais sur des moments en lien avec leur retour, etc. Girtler décrit ce processus d'identification nécessaire au maintien de la proximité comme suite : on gagne en confiance, on élargit l'horizon de son objet de recherche, on acquiert les connaissances en lien avec l'objet étudié (GIRTLER, 2001, p. 108114). Autrement dit la connaissance nécessaire à la constitution de l'échantillon s'acquiert dans la relative identification de la chercheuse avec son objet de recherche. Lorsque cette proximité en vient à devenir si intense au point de biaiser les interactions vécues et observées il en revient aux acteurs, par la stratégie de dialogue, de renégocier les positions comme celle par exemple de la chercheuse en tant que membre toléré du groupe social étudié. La renégociation s'est faite dans le cas de notre étude d'une part en assemblée générale (entretiens directs 
avec le groupe social observé) comme le préconise Girtler (1988, p. 111-113), d'autre part à travers la rédaction de protocoles ouverts sur les dimensions de la proximité ressentie avec le terrain.

Au-delà de mon rôle de chercheuse, mon rôle changea tout au long de mes recherches sur terrain. J'étais parfois la conseillère que les membres de l'association Stern toléraient, quand elles avaient besoin de mes connaissances administratives pour répondre à une lettre ou aller rencontrer des partenaires financiers. Parfois j'étais l'invitée quand nous étions en visite pour saluer un événement privé d'un membre. Parfois j'étais la technicienne aux finances quand l'assemblée me confiait des rapports à lire et à commenter pour permettre à l'association de contrôler et réajuster sa situation financière. Parfois je suis sollicitée comme témoin neutre quand $\mathrm{j}$ 'assistais aux rencontres pour résoudre les crises entre certains membres en conflit. Ces rôles se constituaient selon le quotidien et selon le besoin que les situations au quartier 62 étaient appropriées ou non à laisser place aux positions qui lui étaient assignées (GOLD, 2003). Mon plus grand soulagement fut que depuis le jour de l'aveu, j'étais moi aussi en position d'accepter ou de refuser un rôle que je jugeais incompatible avec mon statut de chercheuse et qui se heurtait à mon éthique. C'est parce que la chercheuse finit par être membre à part entière qu'elle agissait plus comme membre de l'association Stern que comme chercheuse. Elle œuvrait et construisait ses interactions avec les ré-migrantes toutes (chercheuse et rémigrantes) membres et responsables à divers degré d'implication au fonctionnement et à la vie de l'association. D'où les questionnements de la chercheuse dans son courriel adressé à sa superviseure: doit-elle continuer à ouvrer comme membre de l'association? Peut-elle envisager un autre rôle sans briser la relation déjà établie? Cette extrême identification s'est décantée grâce aux dialogues réalisés aussi bien avec la superviseure pour parler de la situation qu'avec les ré-migrantes pour renégocier la proximité dans le moment de recul.

\section{RÉFLEXION AVEC LE TERRAIN - LA CONSTITUTION DES DONNÉES}

En rappel, selon Girtler, ce n'est pas seulement la proximité, mais aussi la distance c'est-à-dire le retrait momentané de la chercheuse hors du terrain qui participe à la production de connaissance que requière l'échantillon pour se constituer (GIRTLER, 2001, p. 78-81). Cette distance selon l'auteur permet de procéder à une réflexion des moments de l'interaction qui sont questionnés parce qu'ils sont irritants, flous, exténuants, conflictuels etc. Ces conflits surviennent lors de l'observation participante parce que selon Girlter l'interaction de la chercheuse avec le terrain se produit dans un contexte d'union de rôles sociaux. L'hypothèse que Girtler sous-tend est la suivante:

Dass das Individuum häufig Handlungen vollzieht, die nur aus Sicht seiner biographischen Identiät verstanden werden können. Dies impliziert auch, dass die jeweilige Bezugsgruppe den Maßtab liefert, mit dessen Hilfe die eigene Stellung in dieser Situation abgeschätzt wird. (GIRTLER, 1980, p. 6). 
[Vu que l'individu la majeure partie du temps interagit de sorte que son interaction ne peut se comprendre que sous l'angle de sa propre biographie identitaire, cela implicite aussi que chaque membre du groupe social lui assigne des marges de manœuvre qui pour eux lui sont appropriées]. ${ }^{6}$

L'approche de Girtler se base sur la tradition phénoménologique de Schütz (1974) et l'articulation des divers cadrages « frames » selon les multiples constructions de la réalité sociale qui composent le cours de l'existence selon Goffman (1991). Girtler comprend donc l'observateur et la chercheuse de terrain comme une union de rôles avec un degré de participation flexible. La flexibilité se joue autant selon les cadrages de la biographie identitaire mise en interaction que selon la situation et le moment in situ. L'un de ces " frames » pourrait être par exemple l'ethnocentrisme (SUMNER, 1906) qui peut conduire à une autre forme de déformation comme limite de la méthode de l'observation participante. L'ethnocentrisme selon Sumner (1906, p. 13) est :

Ethnocentrism is the technical name for this view of things in which one's own group is the center of everything, and all the others are scaled and rated with reference to it.

Définit comme tel, et vu sous l'angle d'approches postcoloniales l'ethnocentrisme est critiqué comme une construction de normes, de valeurs d'un monde social occidental que l'on projette implicitement sur d'autres mondes sociaux non occidentaux lorsqu'on les étudie. Il en résulte des connaissances qui décrivent et, fixent des facettes négatives et racistes de mondes sociaux comme ceux avec une expérience coloniale collective. Cette manière d'observer les phénomènes sociaux, c'est-à-dire étudiés à travers les seules lunettes des idéaux occidentaux, est remise en cause par Abu-Lughod qui, selon notre lecture, plaide plus tôt pour une réflexion du processus de terrain avec une flexibilité vis-à-vis de son ethnocentrisme (ABU-LUGHOD, 2010, p. 425426). Elle propose à travers cette ouverture de déconstruire les dichotomies de l'ethnographie classique (primitif/moderne, dominant/subalterne, développé/ sous-développé, etc.) en les incluant dans notre processus de réflexion de terrain et en les questionnant explicitement tout au long de la recherche (ABULUGHOD, 2010, p. 417-446; NADIG, 1986, p. 11-46).

C'est le plaidoyer pour une flexibilité c'est-à-dire pour une ouverture à la fois vis-à-vis des « frames » de la biographie identitaire que de l'entre-deux. Une telle analyse comme la nôtre noue la perspective de Girtler à celle d'Abu-Lughod dans la même lignée puisque ces cadrages, autrement dit l'ethnocentrisme, entrent dans la construction sociale attendue dans le processus de l'observation participante. L'approche de Girtler suppose que la chercheuse à accepter une union de rôles afin de s'abandonner avec son terrain et celle d'Abu-Lughod complémentairement confère les pistes possibles d'analyse des tensions qui surgissent de cette flexibilité comme sources d'apports de connaissances pour l'étude menée. 


\section{III.1 Les vécus avec le terrain - l'entre-deux des émotions}

L'ouverture à l'hybridité de la chercheuse permet d'analyser les interactions avec la réalité sociale au quartier 62 comme une dynamique qui se laisse interpréter parfois comme une compassion, parfois comme du respect parfois comme de la fierté. Les événements interprétés à travers les données de terrain comme compassion se situent dans les interactions de la chercheuse qui perçoit la réalité sociale d'acteurs qu'elle conçoit comme des ré-migrantes qui subissent le quotidien. Cette compassion se traduit même par moment comme une colère, car à travers ces interactions la chercheuse construit ces ré-migrantes qui subissent à l'image de la femme dominée qui se complaisent dans leur situation. Ces moments de compassion et de colère se manifestent par exemple quand les ré-migrantes se trouvent face à une situation où il s'agit de décider et d'agir en tant que membre de leur association et que certaines en ces moments se retiennent de le faire, car elles doivent d'abord en parler à leurs maris. Ce qui est interprété comme compassion et colère reflète le conflit intérieur de la chercheuse dans sa position de travailleuse/pédagogue sociale face à une situation d'indécision (de son client). Un conflit qui se manifeste à travers la position d'un travail social qui se veut d'accompagner la personne en besoin dans une perspective qui tend par moment plus à l'éduquer et le moraliser (l'aider) qu'une perspective qui tend à comprendre cette personne et à laisser cette personne prendre une décision selon le moment que cette dernière jugera opportun. D'où l'indécision est vécue par la (travailleuse sociale dans son rôle de) chercheuse comme des moments d'incapacité de certaines ré-migrantes d'assumer des décisions.

Par moment aussi nos relations entretiennent des sentiments de fierté et de respect. Par exemple la chercheuse miroitait les ré-migrantes engagées à la recherche d'autres moyens de valoriser leur production d'attiéké comme des actrices créatives pleines de convictions. Ces dernières représentaient pour la chercheuse des ré-migrantes entreprenantes. Les réalités sociales vécues avec les ré-migrantes performantes étaient empruntés d'images de corps résistants à toutes épreuves et de vocabulaires employant des adjectifs valorisants ces dernières:

[...] Elles furent bien accueillantes et apparaissent comme des femmes battantes, mais au fond bien charitables [certaines] avec une voix bien imposante et dominante [...] (Protocole d'observation du 15 juillet 2009).

Les adjectifs battantes, charitables à l'unisson d'une voix féminine imposante et dominante dépeignent bien que la chercheuse perçoit la valeur d'entrepreneurship de ces ré-migrantes.

L'approche par l'entre-deux dans les situations particulières de proximité où les émotions (compassion, colère, respect, fierté) rendaient les interactions floues requiert des moments de distances c'est-à-dire de recul afin de réfléchir sur l'apport de ces émotions dans la constitution des données. J'ai certes mis le focus sur les possibilités d'actions des ré-migrantes, mais mes lunettes d'observation étaient théoriquement biaisées par des principes par exemple 
d'empowerment que je projetais sur les interactions in situ. Ces émotions en référence à ABU-LUGHOD (2010) traduisent également le discours de domination occidentale sur les non-Occidentaux qui résume la relation d'altérité entre le Soi (la chercheuse de terrain avec son savoir occidental) et l'Autre (les ré-migrantes avec leur savoir non occidental).

Lorsqu'à travers les notes de terrain, les protocoles d'observation, le journal de recherche, etc. on repasse en revue la situation avec les ré-migrants de K-dorf, on peut affirmer que la chercheuse projetait sur les interactions, des attentes théoriques répondant plus à ses savoirs et expériences d'hybride qu'à celles du monde social observé.

Pour revenir par exemple à la situation où Samuel m'orientait à travers les chemins, les habitations et les vies de K-dorf, il me confia que la vie, particulièrement pour les ré-migrants qui ont acceptés participer à l'étude, était dure. À cause de l'inondation, les productions agricoles de beaucoup ont été dévastées. Raison pour laquelle continua-t-il je me dois de leur venir en aide, car cela évitera un éventuel blocage de ma recherche. Samuel me manifestait ainsi d'assumer mon rôle d'aidant. Je lui répondis que comme je l'ai souligné lors de l'assemblée à la place du marché de K-dorf, je n'ai pas apporté d'aide et je ne veux en ce sens faire aucune promesse que je ne pourrais tenir.

Samuel fit mine d'avoir compris, mais il était clair qu'il ne l'était pas. Au fur et à mesure il essayait de prendre le rôle de responsable des personnes qui ont accepté que je les rende visite à leur domicile, dans leurs champs de récolte, etc. Il me fit savoir que je pouvais compter sur lui pour convoquer les assemblées. Il se proposait même de contacter, si je le souhaitais, d'autres personnes pour augmenter le nombre de participants. Pendant ce temps j'intensifiais mes contacts individuels avec les ré-migrants en leur téléphonant directement pour fixer les rendez-vous de visite ou confirmer ma participation à des évènements privés ou encore accompagner certains auprès des services sociaux ou administratifs, etc. Deux semaines plus tard après que j'ai passé avec les sept ré-migrants de K-dorf tantôt dans les champs agricoles, tantôt en assistant dans la cuisine tantôt auprès des administrations, etc., j'ai informé Samuel que notre assemblée de dimanche se tiendra sous l'arbre devant sa maison. Quand j'arrivai tous les sept étaient présents et une personne de plus qui connaissait Samuel. J'ai supposé que Samuel voulait l'enrôler dans le processus de recherche sans pour autant m'avoir prévenu. Ne voulant pas le confronter je ne lui posai pas de question au sujet du huitième participant. Je les ai remerciés pour ces deux semaines où ils m'ont accepté et impliqué dans leur quotidien puis j'ai ajouté que sur la base de la confiance et des confidences je ne voulais en ce moment pas rentrer dans les détails. Mais que je suis disposée à rencontrer individuellement ceux qui veulent s'entretenir sur la suite de certains faits qu'on a eu à vivre ensemble. Puis, je leur ai dit qu'ils seraient contactés individuellement par téléphone dans le but de déterminer le moment le meilleur pour la réalisation des interviews. Tanga le plus âgé dit qu'il pense qu'aucun d'eux n'éprouve le besoin d'entamer une conversation sur des détails. Il ajouta qu'on pouvait bien parler de bien d'autres choses et profiter de l'ombre de l'arbre. Il enchaîna en me racontant que leur quartier prépare une 
kermesse. Bien que l'événement va se dérouler dans un mois, continua-t-il, ils présentent que les gens vont passer de bon moment ensemble. Chacun d'eux aborda la conversation autour de la kermesse en vue. C'est sur cette nouvelle que je pris congé d'eux sans que Samuel ne me présente son invité.

\section{III.2 Les stratégies avec le terrain - l'entre-deux des interactions}

Pour analyser la nature de la relation avec les ré-migrants de K-dorf, je rencontrais Roger, ${ }^{7}$ afin d'échanger sur les sens des interactions à travers le matériel écrit (note de terrain, protocoles d'observation, etc.) que j'avais. La prise en compte des protocoles écrits lors de ces échanges de réflexions avec Roger, m'ont permis lors de l'analyse de me rendre compte à quel point Samuel a été un apport pertinent dans le processus de constitution de l'échantillon. Parce que Samuel mettait constamment la chercheuse face aux réalités qui se construisaient à K-dorf en lien avec ses propres attentes à lui, cela amena la chercheuse à se questionner sur la tension qui émanait des situations vécues. Les échanges avec Roger amenaient la chercheuse à comparer et à essayer de toujours trouver une spécificité aux dynamiques des interactions qui se réalisent au quartier 62 et à K-dorf. Cela lui a permis d'interpréter des situations à K-dorf auxquelles participe Samuel comme plus des relations d'adversité que de partenariat. Samuel incarnait à cet effet plus le rôle de l'expert en quête d'aide que celui du ré-migrant. En démontrant son adversité du début jusqu'à la fin de la première phase de terrain, il interpellait la chercheuse dans le rôle de l'aidant autrement dit celui de la chercheuse venue d'Europe comme il la nommait. Samuel participait à sa manière à déterminer les règles du terrain qui armeront enfin la chercheuse à déployer l'aide. En analysant les protocoles d'interaction, je me rendis compte que son interaction a énormément contribué d'une part au renforcement des contours de l'objet d'étude d'autre part à la validation et à la formulation d'autres questionnements afin d'aller en profondeur à la recherche de sens de la relation de partenariat et d'adversité. Le partenariat se construisait avec les acteurs qui assumaient leur rôle de ré-migrants, autrement dit de sujet de recherche et non d'acteurs déterminant les règles de recherche. En tant que ré-migrant Samuel rejetait cependant avec ses interactions d'adversité la condition d'être ré-migrant que la chercheuse projetait sur lui. Il s'efforçait d'imposer tout simplement sa condition d'un homme, habitant de K-dorf ayant besoin d'aide et attendant que la recherche à déterminer ensemble conduise à démontrer la nécessité de cette aide attendue. Cela s'entend que ce n'est ni le terme ré-migrant encore moins la personne de Samuel qui construit cette adversité, mais plus les interactions attendues, voulues, projetées avec ce terme qui nourrissaient les tensions perçues. Les irritations vécues amenaient donc la chercheuse dans les moments de retrait momentané c'est-à-dire de distance à réfléchir sur les sens de ces tensions à travers des questionnements comme par exemple : en quoi consiste la différence entre les ré-migrants et les non-ré-migrants ? Qu'est-ce que l'étiquette être ré-migrant que je projette sur nos interactions implique pour l'étude ? Ce processus d'analyse permit de consolider l'échantillon aussi bien dans son ensemble que dans sa 
spécificité de façon aussi exhaustive que possible. Et ce tout en allant chercher les apports que les cadrages hybridité pouvaient favoriser dans la constitution des connaissances requises et attendues comme données de terrain :

Par moment je pense que les sujets sur lesquelles se porte ma recherche ont besoin d'une travailleuse sociale comme moi puis quand $\mathrm{j}$ 'en viens à m'abandonner pour vivre comment ils arrivent à s'en sortir d'eux-mêmes je me dis : contente-toi tout simplement de les étudier. (Journal de terrain, 31.10.2010).

Ces moments et situations du terrain où la chercheuse se donne des lignes directives de discipline à adopter m'ont conduit également à tenir à ce que la transparence accompagne nos interactions. Aussi je pris le temps à chaque fois que j'avais une interview avec les ré-migrants à les éclairer sur la signification de leur consentement qu'ils doivent signer aussi bien pour la recherche que pour eux en tant que participants. J'insistais sur l'anonymat et la sécurité que j'employais pour protéger leur sphère privée. À y réfléchir, ces moments permettaient à la chercheuse de les rassurer certes, mais de se rassurer soi-même quant à son rôle primaire de chercheuse qui s'assignait à cet effet certains positionnements pour le respect de l'éthique de recherche.

Au-delà de ces moments autant au quartier 62 qu'à K-dorf j'ai continué à rédiger les lettres administratives de l'association Stern, j'ai rempli des questionnaires qui leur étaient adressés venant de certains ministères dont l'association a bénéficié de soutien, avec la présidente, la caissière et certains membres j'ai participé à des congrès où l'association y était invité, j'ai continué à donner des idées quant à l'orientation scolaire des enfants lorsqu'on me le demandait, etc. Par moment le rôle de représentante, de porte-parole des femmes me fut assigné et je les ai assumés en conséquence.

\section{III.3 Les pratiques avec le terrain - l'entre-deux des attentes}

La chercheuse faisait parfois recours à ses cadrages de travailleuse sociale pour renforcer la proximité. Les moments de distance s'avéraient nécessaires pour la chercheuse de l'entre-deux afin de procéder à des réflexions pour réajuster ses positions avec les interactions selon les aléas du terrain :

« Je me sens si proche de ces personnes avec lesquelles je suis concernée et je vis des difficultés pour ne pas perdre mon rôle [de travailleuse sociale, mais aussi de chercheuse] en vue » (Journal de terrain, 3.11.2009).

Autant ce rôle de travailleuse sociale m'aidait parfois sur le terrain pour consolider la proximité autant ce rôle faisait ombrage pour renforcer le flou de l'entre-deux en prenant relativement toute la place de la chercheuse. D'où la crainte de perdre le rôle de travailleuse sociale qui en quelque sorte était le garant de la proximité requise pour rendre le terrain réalisable. À travers les situations décrites et interprétées il en ressort que les moments de tensions surgissaient quand les attentes pour donner suite ou conclure une interaction donnée sont 
projetées vers la chercheuse. Ces attentes sont en lien avec la relation d'aide. Ce qui interpelle des « frames » dans les positionnements requis en lien avec le rôle de l'aidant. Ces moments font resurgir les conflits sous-jacents de l'hybridité (l'entre-deux) de la chercheuse qui se demande si sa position est d'intervenir pour une clientèle par moment si vulnérable, par moment si autonome. Ou doitelle assumer tout simplement son rôle de chercheuse (venue d'Occident certes, mais ayant à la fois un statut social d'origine du Burkina Faso). Ou encore plus complexe celle de chercheuse Burkinabè de retour chez elle, etc.

Ces doutes interagissent selon les marges de manœuvre qui sont appropriées aux cadrages de la biographie identitaire de l'observateur-chercheuse (GIRTLER 1980, p. 6), car ces interactions se trouvent aussi observées et évaluées par les tiers. Et ce comme l'illustre le propos de Marie dans une interview tenue avec elle. Elle me dit que l'association a reçu aussi bien des étudiants burkinabè que des étudiants étrangers, mais aucun n'a été comme moi. Puis elle poursuivit :

[...] c'est pour cela parfois l'autre jour même Fatmata et moi on était en train de dire peut être c'est parce que c'est l'Allemagne puisque nous on a essayé de voir la GTZ qu'on travaille ici comment le personnel de la GTZ par rapport à d'autres services sont là je dis ça c'est une autre éducation qu'elle a eu là-bas et vraiment c'est bien c'est bien la patience prend tout son temps quelque soit ce que la personne est l'aborder là vraiment c'est très bien. (Passage de l'interview avec Marie, 31 octobre 2009).

Marie relate et analyse la manière dont la chercheuse mène son enquête en lien avec les services que le personnel de la GTZ leur offre. Une analyse qu'elle fait après avoir observé les interactions de la chercheuse puis en avoir discuté avec sa collègue, Fatmata. En interprétant les propos de Marie ce qui distingue les interactions de la chercheuse comme elle en est venue à la conclusion avec Fatmata c'est l'éducation qu'elle a eu là-bas (en Allemagne). Elles soutiennent leur hypothèse en faisant ressortir la particularité de l'offre de service d'aide de développement allemand qui se différencie avec les autres services à travers la patience, le temps qu'on investit, l'ouverture vers autrui, etc. On peut sous-tendre que dans les interactions se sont plus les cadrages des acquis professionnels de la chercheuse qui sont visés comme le dit Marie. Autrement dit c'est moins la construction sociale collective en tant que femme, ré-migrante, burkinabè, mais plus l'hybridité de l'expérience académique allemande qui était interpellée. D'où le rôle de coopérant au développement qu'on lui assignait par moment.

Par exemple à K-dorf lors de mes visites individuelles un des ré-migrants me dit qu'il est au courant que les Européens aiment bien les enfants africains et qu'il voulait me demander de lui trouver du parrainage en Allemagne pour ses enfants. Je lui répondis que je ne m'y connaissais pas dans le domaine et que je n'avais aucun contact avec les organisations qui s'occupe de parrainage. Sans lui laisser le temps, j'ai continué à parler en lui disant que je rencontrais pas mal d'affiches en Allemagne montrant des images d'enfant aux prises à la misère en 
Afrique. Au vu de ces affiches, je continuais à lui raconter, on finit par croire qu'en Afrique les enfants sont maltraités, car ils ont des parents incapables de prendre soin d'eux et il en revient aux Européens de s'en occuper. Dans cette situation la chercheuse de nouveau se retrouve transposée dans une relation d'aide. C'est une interaction qui donne de nouveau une lecture de la chercheuse travailleuse sociale qui interpelle son client à compter avant tout sur ses propres potentiels tout en tenant un discours critique vis-à-vis d'un programme qu'elle perçoit sous l'angle postcolonial. Quoi que cette situation permet de discuter les relations d'inégalités sociales cette fois-ci pas celles perçues à travers les conditions d'habitation disparates à K-dorf (zone lotie, zone non lotie) évoquées par la chercheuse pendant la phase d'orientation. Mais plus tôt celle illustrant une inégalité d'accès aux ressources dans une relation de privilège. L'interlocuteur à K-dorf fait référence à la position plus propice de la chercheuse qui compte tenu de ses ressources linguistiques, académiques, sa situation géographique (résidence en Allemagne) et de son statut social d'origine du Burkina Faso est mieux adéquate in situ pour jouer le rôle de l'intermédiaire qu'on cherche à lui assigner dans la construction du projet possible de parrainage.

\section{Conclusion}

La description et l'analyse ethnographique de notre étude éclairent, expliquent et situent les relations complexes de terrain qui par moment sont des interactions en lien avec la recherche de l'amélioration de condition de vie. La chercheuse voulant mener sa recherche avec succès pour son titre académique puis les ré-migrantes et ré-migrants orientant la recherche vers les possibilités où ils peuvent en tirer bénéfice. Par moment nos relations thématisent l'inégalité d'accès aux ressources, parfois elles entretiennent des tensions. Ces dernières venant secouer tous les imaginaires projetés envers les catégories migration et acteurs (sujets, performants, actifs, entrepreneuriat, etc.). Nos actions résumaient aussi une relation d'aide c'est-à-dire une aide pour la réalisation de l'étude, une aide pour que l'étude manifeste simultanément ses bénéfices autant vis-à-vis de la chercheuse que du groupe social étudié, une aide vis-à-vis des projets qui nourrissent le quotidien et le futur des acteurs avec lesquels on interagit. Ce qui conduisait à des tensions requérant des moments de consolidation de la proximité où des moments de retrait momentané à travers la distance. Se sont en somme des interactions qui se négociaient, se nouaient, se dénouaient et se réajustaient dans l'objectif d'offrir la place aux conflits, aux malentendus et parfois aussi aux non-dits pour construire une réalité sociale du moment, de la situation et de l'action qui s'y prêtaient. Dans le cas de la présente étude la distance c'est-à-dire reculer se manifestait lorsqu'on assignait à la chercheuse des rôles faisant appel à ses cadrages les plus flous c'està-dire de son hybridité avec lesquelles elle se retrouve en relation de l'entre-deux (chercheuse, travailleuse sociale, Burkinabè, Ré-migrante etc.). D'où la question n'est pas seulement d'interagir, mais plus comme qui à la place de qui et en tant que qui ? La proximité c'est à dire mieux sauter s'articulait dans les moments où à la fois les rôles de chercheuse de l'entre-deux et de travailleuse sociale dans son rôle de chercheuse trouvaient des instants de cohérence d'action. Ce sont 
par exemple les moments d'aveu, d'entente, d'ouverture, de réajustement et de repositionnement de rôles avec l'interaction. Autrement dit proximité et distance sont en relation et se manifeste par tantôt comme du partenariat tantôt comme de l'adversité, tantôt comme de la compassion, tantôt comme de la fierté. Ce sont ces « frames » à la fois de la chercheuse de l'entre-deux et de la chercheuse en travail social mis en flexibilité et en dialogue qui comptent tout fait nourrissent, construisent et constituent les données recueillies, production des interactions initiées, entretenues et poursuivies in situ.

\section{Notes}

${ }^{1}$ Je remercie Stéphanie Garneau et Dossou Jonas Azonaha pour leurs remarques sur une première version de ce texte.

${ }^{2}$ Le terme ré-migration écrit avec un trait d'union signifie pour notre étude un processus incluant une rémigration c'est-à-dire un retour pour les travailleurs migrants nés au Burkina Faso et une migration pour ceux qui sont nés en Côte d'Ivoire et qui rentrent pour la première fois dans leur pays d'origine pour s'y installer. Dans le contexte burkinabé ces ré-migrants sont connus sous le terme 'rapatriés de la Côte d'Ivoire'.

${ }^{3}$ L'association Stern regroupe des femmes ré-migrantes. L'activité principale de l'association consiste à la fabrication de l'attiéké, une denrée alimentaire quotidiennement consommée en Côte d'Ivoire qui se produit à base de farine de manioc. Le retour des ré-migrants en général et l'activité de ces ré-migrantes en particulier a contribué à faire de l'attiéké une denrée quotidiennement accessible au Burkinabè. Il est à noter que c'est grâce à la divulgation de cette denrée par les ré-migrants que l'attiéké est rentré dans les habitudes alimentaires du quotidien des habitants du Burkina Faso.

${ }^{4}$ Les activités principales des ré-migrantes et ré-migrants de K-dorf sont l'agriculture, le commerce, la pêche, l'élevage. Certains d'entre eux alternent ces activités avec des occupations à temps partiels dans les petites et moyennes entreprises (sécurité, agro-industrie, industrie alimentaire).

${ }^{5}$ Cette expérience d'étudiante étrangère est abordée spécifiquement sous une forme littéraire dans mon roman intitulé La croisée de nos vies (OUEDRAOGO, 2014).

${ }^{6}$ Traduction du sens de la citation par l'auteure du présent article.

${ }^{7}$ Roger a été une personne clé de mes recherches de terrain, car il m'a facilité l'accès avec le terrain et m'a offert des séances d'écoute et réflexions sur les crises, les blocages vécus lors du terrain ainsi que les stratégies pour renouer avec le terrain. Ses connaissances juridiques et celles du contexte social étudié ont participé à la constitution progressive de notre objet de recherche avec le terrain.

\section{RÉFÉRENCES}

ABU-LUGHOD, L. Écrire contre la culture : réflexions à partir d'une anthropologie de l'entre-deux. In : CEFAÏ, D. (Ed.). L'engagement ethnographique. Paris : Édition de l'EHESS, 2010. p. 417-446.

BEAUD, S. ; WEBER, F. Guide de l'enquête de terrain. Paris : La Découverte, 1998.

BECKER, H. Inférence et preuve en observation participante. Sur la fiabilité des données et la validité des hypothèses. In : CEFAÏ, D. (Ed.). L'enquête de terrain. Paris : La Découverte MAUSS, 2003. p. 350-362. 
FLAMANT, N. Observer, analyser, restituer : conditions et contradictions de l'enquête ethnologique en entreprise. Terrain [en ligne], DOI : 10.4000/ terrain2505, n. 44, p. 136-152, 2005.

GEERTZ, C. La description dense : vers une théorie interprétative de la culture. In : CEFAÏ, D. (Ed.). L'enquête de terrain. Paris : La découverte MAUSS, 2003. p. 208-233.

GARNEAU, S. Du terrain proche « en pointillé » à l'immersion sur le terrain lointain : jeux de rôle du chercheur et effets de connaissance dans l'enquête multisituée. In : ROULLEAU-BERGER, L. (Dir.). Sociologies et cosmopolitisme méthodologique. Toulouse : Presses Universitaires du Mirail, 2012. p. 137-154.

GLASER, B. ; STRAUSS, A. La découverte de la théorie ancrée : stratégies pour la recherche qualitative. Paris : Armand Colin, 2010.

GIRTLER, R. Vagabunden in der Großstadt : teilnehmende Beobachtung in der Lebenswelt der Sandler. Wien : Ferdinand Enke Verlag, 1980.

GIRTLER, R. Methoden der qualitativen Sozialforschung. Anleitung zur Feldarbeit. 2. ed. Wien : Böhlau Verlag, 1988.

GIRTLER, R. Methoden der qualitativen Sozialforschung : anleitung zur Feldarbeit. 4. ed. Wien : Böhlau Verlag, 2001.

GOFFMAN, E. Les cadres de l'expérience. Paris : Minuit, 1991.

GOLD, R. Jeux de rôles sur le terrain : observation et participation dans l'enquête sociologique. In : CEFAÏ, D. (Ed.). L'enquête de terrain. Paris : La Découverte MAUSS, 2003. p. 467-615.

GOUlET, J.-G. A. Trois manières d'être sur le terrain : brève histoire des conceptions de l'intersubjectivité. Anthropologie et Sociétés, [S.1.], v. 35, n. 3, p. 107-125, 2011.

LEE, E. S. A Theory of Migration. Demography, v. 3, n. 1, p. 47-57, 1966.

LÉVI-STRAUSS, C. Tristes Tropiques. Paris : Plon, 1955.

MANDÉ, I. Les avatars de l'immigration organisée: le SIAMO, un organisme négrier au service de la Côte d'Ivoire, 1950-1960. In : COQUERY-VIDROVITCH, C. et al. (Éd.). Être étranger et migrant en Afrique du XXe siècle : enjeux identitaires et modes d'insertion. Paris : L'Harmattan, 2003. p. 107-125. Volume I: Politiques migratoires et construction des identités. 
NADIG, M. Die verborgene Kultur der Frau : Ethnopsychoanalytische Gespräche mit Bäuerinnen in Mexiko. Subjektivität und Gesellschaft im Alltag von OtomiFrauen. Frankfurt am Main : Fischer Taschenbuch Verlag GmbH, 1986.

OUEDRAOGO, D. ; PICHÉ, V. Dynamique migratoire, Insertion urbaine et Environnement au Burkina Faso: Au-delà de la houe. Burkina Faso : L'Harmattan Presses Universitaires de Ouagadougou, 2007.

OUEDRAOGO, S. V. Methodisches Vorgehen: interpretative Stratégie. In : . Von Côte d'Ivoire nach Burkina Faso : Re-Migration und Integration Die Perspektive burkinischer Arbeitsmigrantinnen und -migranten. Berlin : Logos Verlag, 2013. p. 86-108. Thèse (doctorat), Université de Kassel, Allemagne, 2013.

OUEDRAOGO, S. V. La croisée de nos vies. Paris : Société des écrivains, 2014.

PACÉRÉ, T. F. Burkina Faso : Migration et Droits des Travailleurs (1897-2003). Paris : UNESCO/KARTHALA, 2004.

SCHÜTZ, A. Der sinnhafte Aufbau der sozialen Welt : eine Einleitung in die verstehende Soziologie. Band II, (édité par Martin Endress et Joachim Renn). Konstanz, UVK Verlagsgesellschaft mbH, 1974.

SUMNER, W. G. Folkways : a study of the sociological importance of usages, manners, customs, mores and morals. New York : Dover, 1906. Disponible in : $<$ https://archive.org/details/folkwaysastudys00sumngoog $>$. Digitized by the Internet Archive in 2011.

WEINBERG, M. ; WILLIAMS, C. Soziale Beziehungen zu devianten Personen bei der Feldforschung. In: FRIEDRICHS, J. (Ed.). Teilnehmende Beobachtung abweichenden Verhaltens. Stuttgart: Ferdinand Enke Verlag, 1973. p. 83-108.

Reçu: Juillet 17, 2014 Accepté: Août 22, 2014 